\title{
Liderazgo y Coaching
}

Angel Martínez Moreno

Universitat de València

\begin{abstract}
$3 \times 10$ *
El propósito de este artículo es señalar algunas técnicas y maniobras de intervención en los procesos de coaching destinados al desarrollo de las capacidades de liderazgo. Para ello se entiende el liderazgo como un proceso colectivo más que individual en el que resultan tan importantes las características y comportamientos del líder como los de los miembros del grupo. En este contexto se identifica el liderazgo transformacional como referencia fundamental de trabajo considerando, tanto las aplicaciones funcionales del mismo, como los posibles efectos perversos que un desempeño deficiente del rol de líder puede generar en los grupos.
\end{abstract}

The aim of this paper is to highlight some techniques and maneuvers intervention in the processes of coaching for the development of leadership skills. This leadership is understood as collective rather than an individual process that are as important characteristics and behaviors of the leader as the group members. In this context, transformational leadership is identified as a fundamental reference for to work in the coaching process, considering, one hand, the functional applications and the other hand the possible perverse effects than a bad performance can generate in the leadership role in groups.

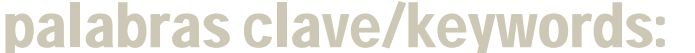 \\ Liderazgo, procesos grupales, coaching, técnicas para la intervención, liderazgo transformacional. Leadership, group processes, coaching, intervention techniques, transformational leadership.}

\section{1.- Introducción}

El estudio del liderazgo constituye uno de los tópicos más relevantes, antiguos y reiterados en el conjunto de las ciencias sociales. En realidad se refiere a un fenómeno que presenta una enorme relevancia: cómo una persona, el líder, puede ejercer la influencia suficiente sobre otras para que modifiquen voluntariamente su comportamiento en la dirección de lo que éste pretende.

También se trata de un fenómeno que ha dispuesto de múltiples perspectivas de estudio que en muchas ocasiones se pueden considerar contrapuestas entre sí.
En realidad se trata de un fenómeno que pudiéramos considerar como natural en el sentido de que puede emerger de manera espontánea, tanto en los colectivos humanos como en los animales que viven en contextos específicamente sociales. En los grupos informales el poder, entendido como la capacidad de influencia sobre la conducta de los otros miembros, se reparte de manera desigual. De hecho, incluso cuando no se identifican jerarquías formales, siempre es posible identificar conductas propias del ejercicio del liderazgo que, en muchas ocasiones, se producen de manera espontánea:

Algunos miembros hacen propuestas más frecuentemente que otros y el resto de los 
componentes del grupo las asumen como ideas estructuradoras de la actividad colectiva.

Algunas personas dan su opinión sin esperar a conocer la de los otros y muchos de estos últimos construyen los contenidos de su pensamiento a partir de esas opiniones iniciales.

- Algunas personas, cuando proponen la adopción de una decisión en una situación de ambigüedad o de riesgo para el grupo, generan mayor sensación de seguridad en los miembros.

- Algunas personas son más buscadas que otras por los miembros del grupo como fuente de reconocimiento.

- Algunas personas son más capaces que otras de generar sinergias entre las capacidades de los miembros de un grupo y de ampliar así el volumen y la entidad de los objetivos y metas colectivas.

- Algunas personas son más aceptadas que otras cuando se trata de mediar en un conflicto entre miembros de un mismo colectivo.

Es a través de estas diferencias como se construye el liderazgo en los colectivos humanos naturales, es decir, en aquellos que no han sido diseñados a través de un proceso formal de construcción ${ }^{1}$.

La llamada psicología evolucionista se ha ido ocupando de forma sistemática del fe-

\footnotetext{
1 Es el caso de las organizaciones de todo tipo, que se crean a través de un proceso de diseño, en la pretensión de que éstas se adapten a los objetivos y que se consideren las capacidades concretas de los miembros. Uno de los elementos del diseño organizativo es, precisamente, la estructura jerárquica, que determinará quién ha de tomar cada tipo de decisiones y en qué circunstancias. Asimismo, se determina quién ha de condicionar la conducta de otros, en qué aspectos y bajo qué circunstancias específicas.
}

nómeno del liderazgo como instrumento de mejora de los procesos adaptativos de las sociedades humanas a entornos exigentes en cuanto al manejo de recursos escasos, y ha propuesto el liderazgo como vinculado a la dominancia y al servicio de una mejor coordinación del grupo (Van Vugt, 2006) aunque, eso sí, desde el planteamiento de que el líder, de una u otra forma, persigue ventajas en la permanencia de sus genes en el colectivo (Gaviria, 2011). Como resulta esperable, desde el punto de vista evolucionista, el liderazgo ha cambiado en algunos de sus mecanismos y contenidos de forma que, por ejemplo, la dominancia física se ve sustituida, en las sociedades modernas, por cuestiones como puedan ser la diversidad de capacidades, el prestigio social, etc. para explicar la emergencia de líderes (Van Vugt, Jhonson, Kaiser, y O'Gorman, 2008).

En los colectivos formalizados (organizaciones, instituciones, equipos de trabajo, etc.) se pretende que los procesos de influencia social se encuentren prescritos a través de roles formales. En la práctica, no siempre se consigue que coincidan los roles formales de poder (jefes) con los roles informales que son generados a través de procesos de interacción (planificados y no planificados) entre los miembros de un colectivo. Las empresas, en concreto, pueden llegar a diseñar planes específicos a fin de conseguir que las personas dotadas de poder formal (jefes) desarrollen capacidades que permitan el ejercicio de la influencia personal sobre sus colaboradores y subordinados directos. Es bien conocido que la influencia informal es, en muchas ocasiones, bastante más eficaz que la formal cuando se trata de evitar y minimizar el efecto negativo de los conflictos, aumentar la cohesión grupal, facilitar la creación de 
sinergias o asumir objetivos exigentes, por ejemplo.

El planteamiento del liderazgo como un proceso de influencia de un individuo sobre otros nos enfrenta a la consideración ineludible de su carácter grupal. De esta manera, el liderazgo no constituye una propiedad que se puede atribuir a una persona específica, sino un proceso que caracteriza la interacción entre el líder y el grupo en que se ejerce la influencia. El liderazgo, en tanto que proceso social, se realiza en grupos concretos y no se entenderá la actuación del líder sin comprender la de los seguidores. En realidad, puede que, más importante que intentar explicar la actuación del líder, quizás sea entender por qué y cómo los seguidores se dejan influir por una persona del grupo (Haslam, Reicher y Platow, 2011).

Sin embargo, el liderazgo no siempre genera efectos positivos. En ocasiones puede anular el pensamiento propio, producir fenómenos de sobreadaptación a las actitudes y opiniones del líder, fomentar el seguidismo acrítico, entorpecer la creatividad, generar dependencia de los miembros del grupo o dificultar la innovación, entre otros efectos no deseados.

La consideración de los aspectos positivos y negativos del liderazgo es más importante todavía si consideramos que constituye un factor de estructuración del grupo que determina lo que éste puede y lo que no puede conseguir y que, además, tiende a perpetuarse. Efectivamente, en la práctica, el liderazgo muestra una clara tendencia a consolidarse en el grupo, de tal manera que constituye un rol que se mantiene en el tiempo y que, cuando se pierde, siempre supone la existencia de un conflicto entre el líder y el resto del grupo o, muy frecuente- mente, implica la expulsión de la persona del colectivo $y$, muchas veces, la pérdida de su prestigio personal junto a la posición jerárquica.

En todo caso, el liderazgo se convierte a menudo en un condicionante, positivo y negativo, del éxito de los procesos de innovación en las organizaciones (y en la propia sociedad) así como un instrumento, no siempre consciente, de desarrollo de los grupos, pero también de las personas en sus múltiples dimensiones, individual, profesional y social.

En el presente artículo se señalan, en primer lugar, los enfoques básicos de consideración del liderazgo como fenómeno psicosocial considerando, asimismo, las versiones ineficaces o perversas del mismo. Posteriormente se describen una serie de técnicas que se utilizan, desde el planteamiento del coaching, para desarrollar la capacidad de liderazgo en personas específicas. $\mathrm{Al}$ respecto ha de tenerse en cuenta que el coaching se diferencia de la formación, el mentoring y de otros métodos de capacitación personal y profesional en que la relación entre el destinatario y el profesional que dirige el proceso no se centra en el trasvase de conocimientos o de habilidades que el formador o mentor posee y el cliente no. Por el contrario, en el coaching se le proponen a la persona situaciones o cambios en la forma de abordar situaciones, analizar procesos o tomar decisiones, sin indicar contenidos concretos, aunque insistiendo en el cuestionamiento sistemático de los métodos y esquemas de trabajo que hasta el momento ha venido desplegando la persona cliente. Así, el coach, en tanto que profesional, no precisa ser más experto o acumular más conocimientos concretos y habilidades que el cliente, sino que ha de 
ser considerado como un especialista en la metodología del cambio para propiciar situaciones capaces de provocar el desarrollo de las capacidades del cliente a través de su propia experiencia. Esta especificidad ha provocado que muchas de las prácticas y aportaciones metodológicas desarrolladas en el ámbito de la psicoterapia, en sus diversas escuelas y perspectivas, y del consueling hayan podido aportar, como se puede comprobar en la exposición de técnicas que se incluye en el presente trabajo, la parte fundamental de la "caja de herramientas" a la metodología del coaching.

\section{2.- Las necesidades grupales a las que responde el líder}

Independientemente de las diferentes formas de abordar el liderazgo, podemos plantear que se trata de un fenómeno psicosocial que, en los grupos humanos, se desarrolla para dar respuesta a una serie de necesidades que comparten los diferentes colectivos casi de forma independiente de su naturaleza (formales, informales, diversos, homogéneos, con mayor o menor grado de cohesión, con objetivos exigentes o laxos, etc.):

\section{- La necesidad de establecer metas del} comportamiento colectivo. Todo grupo necesita saber hacia dónde se dirige y por tanto qué sentido tiene "trabajar juntos" incluso más allá de los requerimientos laborales inmediatos. Por ello el líder ha de contribuir de forma activa y determinante en la definición de la visión del colectivo, grupo o equipo. Definir la visión supone la participación protagonista del líder para propiciar que los miembros de un colectivo sean capaces de imaginar cómo será la realidad cuando se hayan conse- guido las metas pendientes ${ }^{2}$. En la misma dirección, la visión ha de explicar y justificar adecuadamente los contenidos que se proponen y sus ventajas hasta conseguir la adhesión de los miembros, lo que se traducirá en la implicación de estos en las propuestas concretas y esfuerzos requeridos (estrategia) para conseguirla.

Además de las metas, que pueden tener la forma de visión en los sistemas sociales más estructurados, y de objetivos más coyunturales en la mayoría de los colectivos humanos, existen otros factores que contribuyen a articular el comportamiento colectivo. Así, por ejemplo, la necesidad de estructuración del tiempo, de los esfuerzos y de los recursos. Es inherente al líder facilitar a los miembros del colectivo la ordenación de sus esfuerzos haciéndolos complementarios, incluso cuando esa estructuración se limita a fijar los resultados a obtener, permitiendo los máximos niveles de autonomía personal en los procesos de trabajo. Lo que el líder, fundamentalmente, proporciona es sentido, es decir un para qué del esfuerzo de las personas. ${ }^{3}$ El establecimiento de plazos, la especificación de objetivos, la anticipación de recursos necesarios o la identificación de límites, etc., aparecen como un mecanismo estructurador de primer orden que dota de seguridad colectiva y de sentido estratégico a los esfuerzos de las personas.

\footnotetext{
${ }^{2}$ Un ejemplo clásico de visión es la que planteó Martin Luther King cuando en uno de sus más conocidos discursos comenzó con "I had a dream..." y que describe como será una sociedad sin discriminación racial.

${ }^{3}$ Es bien sabido que el esfuerzo sin objetivo no se mantiene en el tiempo, ni individual ni colectivamente. "Los niños corren mejor si lo hacen detrás de una pelota"
} 


\section{- La necesidad de disponer de modelos} de comportamiento. Los miembros de un colectivo comparten ciertos valores y actitudes en cuestiones concretas. Los valores se traducen en conductas que han de ser realizadas por alguien de manera demostrativa. El líder se convierte en el referente de realización de esas conductas. De hecho, en muchas ocasiones, los valores no preceden a las conductas sino al contrario, los miembros del colectivo deducen la existencia y adecuación de determinados valores al interpretar la conducta de sus líderes.

Este hecho nos ofrece una idea de la responsabilidad que asumen los líderes en las organizaciones, los grupos y equipos de trabajo: con su comportamiento están conformando los valores (y consecuentemente las prácticas de trabajo y de relación) de los miembros del grupo, sobre todo cuando éste se encuentra en los momentos iniciales de su definición.

En otros momentos, cuando se trata de grupos muy consolidados, el comportamiento, "original" o incoherente respecto a los valores asumidos, por parte de un nuevo líder puede generar fuertes reacciones de rechazo a su liderazgo. El líder ha de representar al grupo y sus valores, constituyendo un factor de identidad del mismo tal como en su momento planteó la Teoría de la Identidad Social (Tajfel y Turner, 1979) y, en ese sentido, el líder es modelo para el grupo pero también es percibido por los miembros como representación del mismo.

- La necesidad de que la responsabilidad se identifique con una persona concre-

ta. En situaciones que requieren la toma de decisiones que supuestamente comportarán consecuencias graves y que ge- neran incertidumbre, los grupos humanos tienden a centralizar la responsabilidad en una persona que la asume de manera individual aunque cuente con el apoyo explícito del conjunto de los miembros del grupo.

Es precisamente en momentos de crisis, que han requerido la toma de decisiones arriesgadas, cuando han emergido muchos líderes que posteriormente han podido consolidar su rol en situaciones más normalizadas. Sin embargo también en esas situaciones de crisis se han desprestigiado líderes que se encontraban aparentemente consolidados, solo por no haber sido capaces de asumir responsabilidades individualmente y haber pretendido diluirlas en el conjunto del grupo.

- La necesidad de disponer de una instancia de autoridad capaz de resolver los conflictos. Los colectivos humanos y las organizaciones no pueden, ni probablemente resultaría útil, identificar todas las posibles situaciones de conflicto que pudieran generarse entre los miembros de un grupo. Así pues no todo se puede anticipar para poder dotarse de reglamentaciones explícitas que prevean los posibles conflictos interpersonales o entre grupos de intereses. Por ello, es necesario que alguien, a quien se le atribuye una autoridad consensuada y aceptada por todos, aborde la resolución de conflictos interpersonales cuando estos alcanzan un determinado nivel de significación y relevancia.

Cuando el líder no asume la función mediadora o de arbitraje en momentos en que es demandada por el grupo, sin una buena razón comprensible por los miembros del colectivo, su liderazgo tiende a diluirse y el consenso respecto a su au- 
toridad disminuye. De la misma manera muchos líderes informales han emergido al ocuparse de mediar en conflictos interpersonales entre miembros del grupo y consolidarse así como un referente de autoridad para el conjunto.

Además de dar respuesta a estas cuatro necesidades de los colectivos es necesario tener en cuenta algunas otras características psicosociales del liderazgo que nos ayudarán a entender en profundidad el fenómeno.

En este sentido podemos considerar que el liderazgo, aunque en muchas ocasiones se presenta como un proceso de influencia que va desde el líder hacia el grupo, constituye en realidad un proceso de influencia recíproca. Los miembros de un colectivo, como ya señalaron Napier y Gershenfeld (1987) emiten señales que son percibidas por el líder acerca de cuáles son las preferencias de los miembros del grupo para ser influidos, así como cuáles han de ser los límites admisibles de esa influencia. Las características del grupo determinan el tipo de liderazgo y las conductas que serán aceptadas y tendrán éxito.

Para comprender el liderazgo en sus aspectos más concretos y operativos no es un asunto menor considerar la capacidad de refuerzo que ejercen los miembros del grupo sobre el líder, a través de procesos de aprendizaje social que van definiendo el rol e integrándolo en el conjunto de roles del colectivo.

Así pues, el liderazgo constituye un fenómeno relacional que encuentra en la reciprocidad y en la complementariedad de roles algunas de las variables explicativas de su dinámica funcional, además de otras variables moduladoras que tienen que ver entre otros aspectos, con los contextos or- ganizativos en los que se desarrolla o los contenidos culturales del entorno.

\section{3.- Los tipos de liderazgo}

Las formas más tradicionales y clásicas de consideración del liderazgo lo planteaban como una característica que poseen algunas personas "especiales" en función de la cual su capacidad de influencia sobre el grupo se magnifica. Estos planteamientos, generalmente conocidos como modelo del "gran hombre" fueron deviniendo en las primeras formulaciones del liderazgo carismático que desarrolla especialmente Weber y a partir de cuyas presuposiciones se multiplican las propuestas para encontrar rasgos de personalidad que se asocien de alguna manera al carisma necesario para ejercer el liderazgo. Puede decirse que actualmente, aunque nadie niega la existencia de personas "especiales", se considera que se trata de fenómenos residuales y excepcionales alejados de las preocupaciones e intereses que son relevantes para las ciencias sociales en su intento de desarrollar modelos de liderazgo capaces de amplificar sus ventajas y de minimizar los posibles efectos perversos.

Desde un planteamiento que se ha venido a llamar Transaccional, Hollander (1978) planteó que el liderazgo constituye un rol que asume un individuo del grupo a través de múltiples episodios de interacción con los otros miembros del grupo en los que se producen intercambios y transacciones entre personas ${ }^{4}$. Desde esta propuesta se

4 Este planteamiento se basa en las Teorías del Intercambio Social que fueron desarrolladas entre otros por Thiebaut y Kelley o por Homans entre otros. Pueden encontrarse las referencias fundacionales en obras de estos autores como por ejemplo: Thiebaut, J.W. y Kelley, 
plantea que el líder es capaz de aportar al grupo:

- Estructura y posibilidades de generar y desarrollar normas que regulen la actividad colectiva.

- Posibilidades de obtener mayores satisfacciones por parte de los miembros del grupo al conseguir resultados y reconocimiento.

Pero también el grupo influye en el líder a través de:

- Concederle un mayor estatus y reconocerle en esa posición superior.

- Ofrecerle la libertad para innovar y hacer propuestas desde sus preferencias personales.

- Permitirle establecer las condiciones para que pueda influir en los otros.

- Proporcionarle información y feedback acerca de su actuación como líder.

En todo caso, asumir la influencia del grupo sobre el líder se relaciona también con los requerimientos de comportamiento del líder que pueden ser considerados como exigencias para que alguien desempeñe el rol con eficacia en un colectivo concreto. Estas exigencias se relacionan directamente con la capacidad del líder para resolver las necesidades del grupo que se han planteado anteriormente.

En una perspectiva diferente al Liderazgo Transaccional, centrado en el intercambio entre el líder y los miembros del grupo, Bass (1990) y Bass y Avolio (1994) han descrito otro tipo de planteamientos que se conceptualizan como Liderazgo Trans-

H.H. (1959) The Social Psychology of Groups. Wiley. New York. Homans, G.C. (1961): Social Behavior: Its Elementary Forms, Harcourt, Brace \& Worl, New York. formacional y que se centran fundamentalmente en que el líder:

- Pretende modificar la perspectiva en la que los miembros del grupo perciben sus roles y el contenido de su actividad, estimulándoles para que generen cambios en sus actitudes.

Intenta hacer conscientes a los miembros del valor de los objetivos colectivos para cada una de las personas y para el conjunto.

- Se plantea como un objetivo de su rol facilitar el desarrollo de las capacidades y potencial de cada uno de los miembros del grupo.

- Establece las condiciones para que los miembros se motiven vinculando los objetivos individuales a los objetivos colectivos.

Se propone objetivos especialmente exigentes que suponen una implicación mayor de la habitual por parte de los miembros del grupo.

- Se ocupa del equilibrio emocional de las personas así como de que obtengan un grado de satisfacción elevado, considerando que ello potenciará la motivación y el rendimiento.

Como puede deducirse con facilidad, el Liderazgo Transformacional requiere perfiles de personalidad específicos y habilidades y competencias sociales muy desarrolladas en los líderes. Por ello se ha planteado que este tipo de liderazgo sólo pueden desempeñarlo personas que han aprendido a ser carismáticas (aunque no en el sentido excepcional de los planteamientos clásicos del liderazgo carismático vinculados a la idea del "gran hombre") y que aparecen ante sus grupos de referencia como indivi- 
duos singulares capaces de generar adhesiones que, en muchas ocasiones, son acríticas y que se plantean como el resultado de procesos de adoctrinamiento o incluso, en situaciones más extremas, de dependencia emocional.

Con todo, el liderazgo transformacional parece mostrar una buena capacidad de generar resultados en situaciones de alta exigencia y en grupos muy cohesionados. En la práctica, la pretensión de "transformar" a las personas influidas por el líder no puede decirse que se consiga de manera sistemática, y ello es más cierto cuando se considera que, en muchas ocasiones, los cambios personales no se mantienen después de que haya acabado la situación en la que se produjo la influencia directa del líder (House y Aditya, 1997).

En un intento de superar el contexto y alcance del liderazgo transformacional, se planteó lo que se ha venido a llamar superliderazgo. La idea básica de este enfoque consiste en ir más allá de la potenciación de las capacidades de los miembros del grupo, tratando de generar en éstos las competencias que permitan la autodirección o, como se ha denominado también, el autoliderazgo.

La autodirección supone siempre la liberación de algún grado de dependencia que siempre, inevitablemente, existe cuando se sigue a un líder y consiste, fundamentalmente, en ser capaz de generar el grado de disociación suficiente para que permita que la persona asuma toda la responsabilidad tanto de sus acciones como de sus resultados, la autoobservación, autoevaluación, autorrefuerzo y que desarrolle la capacidad de establecer estrategias eficaces que permitan la entrada en procesos de automotivación (Manz y Sims 1993).
Puede decirse que el superlíder es, en realidad, aquel que no sólo se muestra eficaz en cuanto al logro de resultados por parte de sus seguidores, sino que convierte a estos en líderes de sí mismos y de sus grupos de referencia. Para ello es importante tener en cuenta algunas de las características más relevantes del superlíder:

La propia persona ha de ser un autolíder. Para ello ha de disponer de estrategias cognitivas y de autogestión emocional que le permitan orientarse a sí mismo en los espacios de ambigüedad y resolver los problemas de dependencia emocional o de resolución creativa de problemas.

- Cuando ejerce el liderazgo utiliza la "transparencia metodológica"5 es decir que muestra (o deja que se muestren) cuáles son los mecanismos a través de los cuales se automotiva, aborda el análisis de situaciones complejas, toma decisiones, afronta conflictos, etc., a fin de que los seguidores lo aprendan.

- Genera con sus seguidores patrones de pensamiento, de análisis de situaciones, de afrontamiento de conflictos, etc. que cada uno adapta a sus características y preferencias para facilitar su autogestión.

- Propicia que cada uno de sus seguidores disponga de sus propias metas y objeti-

5 La transparencia metodológica constituye una importante herramienta de aprendizaje, sobre todo cuando se trata de que se incorporen contenidos difícilmente explicitables en forma de procedimientos de conducta o de algoritmos. Se trata de incidir más en el cómo se hacen las cosas que en el qué y, sobre todo, en realizar un esfuerzo por mostrar los procesos internos no visibles externamente de manera directa, que generan resultados visibles. Así, por ejemplo, es importante identificar el proceso mental de toma de decisiones, o los procesos de exploración de posibles soluciones a problemas difíciles o ambiguos en su formulación. 
vos además de los que pertenecen al colectivo.

- Potencia una cultura del autoliderazgo y la autogestión a través de reforzar continuamente las conductas coherentes con la autonomía de los seguidores.

- Utiliza el equipo como marco cultural en el que se potencia la autorresponsabilidad de los miembros. De esta forma lo grupal no se convierte en refugio de la falta de autonomía sino en un entorno que la intensifica.

\section{4.- Modalidades disfuncionales de liderazgo}

Kets de Vries y Miller (1984) han identificado formas de liderazgo que califican como neuróticas para señalar la influencia negativa de algunas características de personalidad de los líderes. Plantean dos perfiles referenciales típicos:

\section{a) El líder histérico}

Se trata de una persona que, en primera instancia, se presenta emocionalmente atractiva para los otros y a la que, por tanto, le resulta fácil atraer la simpatía inicial de los demás. Generalmente goza de popularidad tanto en su organización como en las organizaciones de su entorno. Sin embargo se trata de una persona vulnerable emocionalmente que requiere el cultivo permanente de su imagen. Es capaz de iniciar muchos proyectos incorporando a otras personas que asumen riesgos pero en muchas ocasiones no consigue finalizar los proyectos.

Entusiasma a sus colaboradores pero no sigue los proyectos y no administra adecuadamente los recursos si ello implica un trabajo sistemático y metódico. Puede presentar cierta tendencia a la megalo- manía y asume riesgos excesivos.

\section{b) El líder obsesivo}

Responde al prototipo de un apersona socialmente inhibida y poco imaginativa, se muestra habitualmente muy responsable, disciplinado y laborioso. Asimismo, suele manifestar una moralidad estricta en su comportamiento con los demás.

Se mueve bien en ambientes estables en los que el respeto a procedimientos establecidos y repetitivos constituya una garantía de éxito. Desconfía de las novedades y prefiere aquellos modos de hacer que ya han demostrado su eficacia repetidas veces en el pasado. Suele ser un administrador estricto de los recursos disponibles y planifica con criterios de eficiencia. Planifica al detalle y mantiene relaciones jerárquicas muy rígidas

\section{5.- Coaching y desarrollo del liderazgo.}

Los procesos sistemáticos de coaching en general pretenden potenciar los aspectos vinculados a formas de liderazgo próximas a los planteamientos transformacionales sin por ello renunciar a la complejidad inherente a todos los procesos grupales. Esta complejidad se hace mayor cuando se trata de procesos trasversales, es decir, que se producen al mismo tiempo que el grupo desarrolla sus tareas y ha de responder a presiones tanto del intra grupo como a las provenientes del exterior y que han de traducirse, en muchas ocasiones, en resultados materiales y objetivos.

Algunos autores han propuesto que la perspectiva del coaching implica un nuevo paradigma del liderazgo (Román y Fernández, 2008) que plantea que el líder se incorpora como un agente activo para pro- 
fundizar en los procesos de cooperación y el logro de resultados a partir del compromiso y de la construcción de nuevas estructuras organizativas que cuestionen las tradicionales. Esto, habitualmente, acaba siendo más cierto cuando nos referimos a las intenciones declaradas del profesional del coaching que a la realidad efectiva del trabajo en las empresas. La práctica de múltiples profesionales del coaching en las organizaciones laborales nos muestra como en la mayoría de las ocasiones la demanda de coaching pretende reforzar paradigmas tradicionales de ejercicio de la influencia social y del poder. En general la demanda, quizás desgraciadamente, se mueve en la dirección de conseguir hacer de manera más efectiva lo que ya se venía haciendo. Esta situación no es impedimento para considerar que el coaching puede asimismo constituir una buena herramienta para plantear la incorporación de nuevas formas de ejercicio del liderazgo, pero como lo sería también el entrenamiento tradicional en habilidades directivas.

Hasta ahora hemos visto como la influencia del líder puede ser ambivalente de manera que puede considerarse, por una parte (1) la capacidad para anular a los colaboradores y homogeneizar las formas de pensamiento del grupo haciendo disminuir la capacidad de innovación buscando, a cambio, la efectividad inmediata en la consecución de objetivos concretos; y por otra parte (2) la capacidad para facilitar el desarrollo de procesos de transformación de las capacidades de las personas en las que se influye, de forma que éstas amplifiquen sus posibilidades de obtener mejores resultados, tanto para el grupo como para sí mismas.

Si nos centramos en el segundo aspecto, aquel que se refiere a la potenciación de las capacidades de los colaboradores, podemos identificar tres tipos de acciones que el líder puede realizar de manera sistemática y que se potencian especialmente a través de los procesos sistemáticos de coaching para el liderazgo:

\section{1.- Conversión de recursos en capacidades $^{6}$}

Facilita que las personas identifiquen sus propios recursos personales. Estos están constituidos por una diversidad de componentes: conocimientos, habilidades, hábitos, experiencias pasadas, etc. Cuando los recursos personales se ponen al servicio de objetivos concretos se convierten en capacidades.

El líder, a este respecto, puede hacer que la persona y el grupo tomen consciencia de sus recursos de manera que, al tenerlos presentes, se puedan plantear objetivos más ambiciosos, sintiéndose capaces de conseguirlos. Téngase en cuenta que las personas no siempre son conscientes del potencial de acción de que disponen. Ello supone que, en muchas ocasiones, se propongan objetivos que se encuentran muy por debajo de las capacidades reales que podrían utilizarse.

\section{2.- Establecer las condiciones que permitan el atrevimiento de abordar objetivos de reto.}

En ocasiones las personas desconfían de sus capacidades cuando han de aplicarlas en condiciones que les resultan nue-

6 La teoría de los "recursos y de las capacidades" constituye un referente muy relevante en la economía de la empresa. Lógicamente se trata de otro ámbito de aplicación de los conceptos, aunque se vinculan cuando el liderazgo se plantea en el contexto de la empresa porque permite asumir objetivos más ambiciosos con los mismos recursos de partida. 
vas o cuando implican una modificación importante en las maneras y criterios de afrontamiento de problemas o en situaciones de cambio.

En este sentido, la función del líder es la de incitar a la persona para que asuma riesgos controlados, porque sin ellos la innovación no es posible y el desarrollo personal se convierte en conformidad, permaneciendo ociosos los recursos disponibles para la persona y el grupo.

\section{3.- Protección y feedback ante los errores y fracasos}

$\mathrm{Si}$ se asumen riesgos es porque los fracasos son posibles y quien se atreve también se arriesga. Es importante la experiencia, pero más importante es el aprendizaje que se obtiene de ella. Es por esto que cuando se fracasa resulta fundamental que alguien apoye el proceso de elaboración de conclusiones y de obtención de contenidos de aprendizaje.

En este sentido el líder puede actuar como un referente capaz de absorber parte de la ansiedad que produce el fracaso, al mismo tiempo que objetiva el proceso de evaluación de las causas que lo generaron, potencia la obtención de aprendizajes y facilita que la persona o el grupo insista en la obtención de resultados.

En los procesos de coaching se considera el liderazgo como un tipo específico de interacción social. El objetivo, por tanto, se sitúa en la localización y desarrollo de las capacidades del individuo para optimizar su actuación en los procesos de influencia de manera sistemática. Podemos decir que el coaching para el liderazgo constituye un ámbito de aplicación de conocimientos aportados fundamentalmente por la psicología y que provienen de diferentes áreas de la misma, sobre todo de la psicoterapia, de psicología del aprendizaje y de la psicología social.

La psicoterapia constituye uno de los antecedentes referenciales del coaching en la medida que le proporciona herramientas potentes que, adecuadamente adaptadas, se muestran eficaces en relación a la fijación de objetivos de cambio psicológico, en la modificación de las perspectivas de percepción de la realidad y en el posicionamiento que las personas asumen ante la misma, así como a la gestión emocional que implica tener que afrontar situaciones nuevas, en muchas ocasiones ambiguas, y en las que la frustración y sus consecuencias juega a menudo un papel relevante.

La psicología del aprendizaje permite armar metodológicamente los procesos de incorporación de nuevas habilidades y competencias personales y de gestión psicosocial de forma que éstas se consoliden y se conviertan en hábitos de gestión emocional, cognitivos y de conducta. Ha de considerarse que, en el coaching, más importante que incorporar una nueva habilidad o conocimiento concreto es que se modifiquen las pautas de pensamiento, de gestión de las emociones y del comportamiento a fin de que se establezcan nuevos hábitos y pautas que faciliten la innovación y disminuyan la reiteración acrítica.

Finalmente, y de manera especial en el caso del liderazgo, la psicología social aporta un corpus teórico consolidado que permite identificar los elementos claves que determinan los procesos de influencia social, tanto desde las propuestas de la persona que pretende desarrollar de manera óptima sus roles vinculados al liderazgo como desde el conocimiento de las opciones y condicionantes de respuesta de las personas del grupo o colectivo. 


\section{6.- Algunas técnicas específicas que se utilizan habitualmente para el desarrollo de las habilidades de liderazgo en procesos sistemàticos de coaching.}

Se señalan en este apartado una serie de técnicas y aplicaciones prácticas que han demostrado a lo largo del tiempo, y en diferentes contextos, su capacidad para facilitar el desarrollo de las potencialidades de las personas en su desempeño como líderes. Se plantea aquí un modelo de liderazgo cercano en sus propuestas a la perspectiva del liderazgo transformacional. El efecto de las técnicas se consigue, bien porque facilitan la generación de esquemas de pensamiento alternativos y permiten la innovación en las formas de pensar, o bien porque facilitarán desbloquear hándicaps que impiden el aprovechamiento de las competencias y recursos disponibles, además de potenciar el vínculo de confianza entre los colaboradores y el líder.

Las diferentes técnicas que se proponen, a modo de muestrario, proceden de distintas tradiciones y posicionamientos teóricos que resultarán, para el lector profesional, fácilmente reconocibles. En todo caso, no es difícil identificar cuatro procedencias que han influido de manera especialmente destacada en el coaching en lo referente a la aportación de un arsenal suficiente de técnicas y métodos de trabajo. Básicamente se trata de la perspectiva Cognitivo-Conductual, la Programación Neurolingüística, El Análisis Transaccional y la Gestalt.

Las diferentes propuestas de técnicas, convenientemente adaptadas a situaciones no clínicas, se han convertido en habituales de los procesos sistemáticos de coaching, aunque los profesionales no psicólogos, en mu- chas ocasiones, pueden tener dificultades para identificar claramente su procedencia. Así, el enfoque Cognitivo-Conductual ha propuesto técnicas vinculadas a la identificación de sesgos cognitivos y de valoración, a la reestructuración cognitiva, o a la fijación de contratos de cambio (esta última compartida con el Análisis Transaccional). La Programación Neurolingüística (PNL) aporta esquemas de trabajo vinculados a la consideración del tiempo en los procesos de cambio, las posiciones de observación de los diferentes protagonistas de situaciones diversas, etc. El Análisis Transaccional (AT) se encuentra en el origen de las técnicas que identifican pautas negativas de comportamiento interpersonal como son los "juegos psicológicos" y, en general, los métodos vinculados con la gestión de los diálogos internos que permiten diferenciar entre distintas "partes de la personalidad" que son capaces de hablar entre si y de debatir, tanto en los proceso de análisis de situaciones como en la toma de decisiones. Finalmente, el enfoque de la Gestalt tiene una incidencia especialmente relevante en aquellas técnicas vinculadas a la toma de consciencia de la posición y roles de un individuo en relación a situaciones complejas, así como en la identificación de los mecanismos de autosabotaje, la consciencia del propio cuerpo, o la identificación y gestión de las emociones, por ejemplo.

A modo de ilustración describimos a continuación algunas de las técnicas más utilizadas en el coaching orientado al desarrollo del liderazgo:

\section{1.- Gestión del contrato psicológico.}

El contrato psicológico está constituido por las expectativas que cada una de las partes que conforman una relación 
(en este caso, líder y seguidores). Estas expectativas se refieren a cómo se comportará la otra parte en cada una de las circunstancias previsibles. Cuando las expectativas no se cumplen, porque no se alcanza el desempeño estimado, se produce insatisfacción y decepción con la conducta del otro y se debilita el vínculo relacional. Cuando la actuación del otro supera las expectativas positivamente se genera satisfacción con el otro y se refuerza el vínculo relacional.

Cuando las expectativas se cumplen en la proporción esperada, la percepción resultante es de fiabilidad y consistencia.

El problema es que estas expectativas son subjetivas y no siempre responden a la realidad o a las capacidades efectivas de la otra parte. Es por esta razón que el líder eficaz se ocupa de conocer las expectativas de los seguidores $\mathrm{y}$, en el caso de que considere que no puede cumplirlas, explicita su visión de la realidad y de las posibilidades a fin de que las expectativas se reformulen de manera más realista y se produzca la percepción de fiabilidad y consistencia.

\section{2.- Superposiciones.}

Pequeñas partículas del lenguaje pueden generar efectos perceptivos poderosos en la valoración de situaciones, objetivos y obstáculos. Veamos el efecto de tres partículas especialmente relevantes a estos efectos:

- Este objetivo es muy importante PERO es muy difícil de conseguir

- En este caso la segunda parte de la frase (es muy difícil) acaba siendo más relevante que la primera (este objetivo es muy importante)
- Este objetivo es muy importante $\boldsymbol{Y}$ es muy difícil

- En este caso las dos partes de la frase se mantienen en un plan de igual relevancia

- Este objetivo es muy importante AUNQUE es muy difícil

En este caso la primera parte de la frase (es de muy buena calidad) se realza frente a la segunda (es muy difícil)

\section{3.- Posiciones de observación.}

Una misma situación puede ser percibida de diferentes formas en función de la posición desde la cual se observa. Esta técnica no pretende enseñar cuál es la mejor posición de observación, sino constatar que las diferentes percepciones son complementarias entre sí y que por tanto han de tenerse en cuenta antes de considerar una situación como suficientemente analizada.

\section{La posición 1:}

Corresponde a la percepción de la realidad desde la persona que habla. El individuo ha de esforzarse en mostrar de la forma más exhaustiva la realidad tal como es percibida por él y describirla poniendo en un primer plano sus objetivos, sus intereses, sus formas de reaccionar, dando preferencia a lo propio sobre lo que pueda plantearse desde cualquier otra posición

\section{La posición 2 :}

Para situarse en la posición 2 es necesario colocarse en la perspectiva del otro, ver la realidad como la vería el otro, considerar las situaciones desde los objetivos y los intereses del otro, atendiendo especialmente a cómo se ven las cosas desde su punto de vista. ¿Cómo se me ve a mí desde ahí? Puede ser útil para utilizar adecuadamente 
la posición 2 considerar las condiciones de vida, las rutinas, las costumbres, las presiones a las que se ve sometido, las actividades, etc. a través de descripciones que serán más eficaces cuanto más exhaustivas.

\section{La posición 3:}

Se trata de la perspectiva que adoptaría un observador externo, un espectador que contemplara lo que hacemos como si lo visualizara en una pantalla de cine. Esta posición pretende ofrecer una perspectiva objetivada de la realidad.

El empleo de las tres posiciones perceptivas resulta especialmente útil cuando hay que tomar decisiones que afectan a otras personas, o en situaciones de conflicto interpersonal. El manejo de las tres posiciones permite comprender las actuaciones de los otros y contemplar con mayor realismo y objetividad situaciones que, en un momento dado, pueden estar excesivamente cargadas de componentes emotivos que distorsionan nuestra percepción.

\section{4.- La línea del tiempo.}

Se trata de una técnica que muchas personas utilizan de forma espontánea. Consiste en preguntar: ¿cómo evaluarás esta situación o esta decisión que vas a tomar dentro de 2 ò 3 años?

La línea del tempo permite también otros usos que tienen como objetivo la proyección y la generación de "visiones" en las que el individuo pueda reconocer las consecuencias de posibles decisiones que tome en un momento dado. Así, por ejemplo, puede ser útil preguntar: ¿cómo serán las cosas en tu vida profesional dentro de 5 años si todo va bien? ¿y si todo va mal?
La técnica aporta un gran potencial en procesos de clarificación de valores personales y profesionales.

\section{5.- Reencuadre / Reformulación.}

El reencuadre constituye una técnica que pretende ofrecer una visión diferente a la que el sujeto plantea como problemática a partir de ofrecer un punto de vista distinto o de acentuar un aspecto que la persona no ha considerado o que ha dejado en un segundo plano descontando así su relevancia.

Quizás la mejor manera de explicar el reencuadre sea a través de ejemplos:

Esta idea es imposible se convierte en ¿Qué habría que hacer para que pudiese conseguirse esto?

Esto es muy caro se convierte en $¿ D e$ qué manera podemos conseguir el dinero para comprar esto?

Las posibilidades de fracaso se convierten en posibilidades de éxito

\section{6.- Fragmentación.}

La técnica de la fragmentación consiste de ubicar una determinada experiencia o afirmación en un contexto mayor o menor, más específico o más amplio. Así diferentes experiencias no tienen sentido en sí mismas sino que han de ser comprendidas en un marco que las contenga $y$ del que formen parte.

De esta manera, por ejemplo, cuando alguien plantea que es socialmente inhábil podemos fragmentar esa afirmación planteando en qué se traduce en la práctica esa inhabilidad social:

En qué concretamente no sabe iniciar conversaciones con desconocidos. 
En qué concretamente no sabe responder cuando alguien se dirige a él.

En qué concretamente no sabe contar anécdotas graciosas.

En qué concretamente no sabe fingir que se alegra de ver a alguien, etc.

La fragmentación descubre formas ocultas de generalización y la existencia de estereotipos y prejuicios destructivos

\section{7.- El cuestionamiento de los cuantificadores universales.}

En el lenguaje cotidiano está muy extendido el uso de cuantificadores universales: TODOS, NADIE, SIEMPRE, NUNCA. Se utilizan de forma abusiva y constituyen unos importantes instrumentos de enmascaramiento de la realidad y de debilitamiento de las potencialidades personales.

Piénsese en las siguientes frases:

NADIE me escucha

TODOS me rehúyen

SIEMPRE me pasa lo mismo

NUNCA consigo que tengan en cuenta mi opinión

La fórmula para desactivar los cuantificadores universales es generalmente sencilla, de manera que muchas veces es suficiente con poner el cuantificador entre interrogantes:

$$
\begin{aligned}
& \text { ¿NADIE? (te escucha) } \\
& \text { ¿TODOS? (te rehúyen) } \\
& \text { ¿SIEMPRE? (te pasa lo mismo) (¿de } \\
& \text { entre cuántos intentos?) }
\end{aligned}
$$$$
\text { ¿NUNCA? (consigues que tengan en }
$$$$
\text { cuenta tu opinión) }
$$

En ocasiones los cuantificadores se camuflan en formas casi ocultas pero muy destructivas. Es lo que ocurre cuando después de que alguien haya cometido un error se le dice: eres un estúpido. La desactivación de estos mensajes es difícil y costosa: ¿siempre eres un estúpido?, ¿en qué ocasiones has hecho algo que indique estupidez?, ¿qué has hecho últimamente que no pueda ser considerado estúpido?

\section{8.- Establecimiento de objetivos.}

Uno de los principales servicios que un/a asesor/a puede prestarle a su cliente es ayudarle de forma eficaz a fijar sus objetivos. En este sentido será importante que se consideren algunas características de los objetivos cuando estos se encuentran bien formulados:

Describir con claridad la situaciónresultado: conseguir un objetivo supone construir una situación diferente de la de partida. A veces la persona puede desear el objetivo pero no siempre la situación que se deriva de su consecución.

Los objetivos han de constituir un reto pero han de ser realistas: si no son alcanzables, generan frustración y provocan el abandono y la desmotivación. Si son demasiado fáciles de conseguir, se pierden oportunidades.

Los objetivos han de ser verificables: aquello que no se puede comprobar y constatar carece de sentido. El individuo ha de ser capaz de describir cómo sabrá que ha conseguido su objetivo.

Es necesario determinar previamente el grado de autonomía (capacidad de decisión) con que contará quien asume la responsabilidad de conseguir un objetivo: Es decir, definir hasta qué punto conseguir el objetivo depende de la persona 
o más bien de lo que hagan otros. En este último caso será necesario revisar la formulación del objetivo

Un objetivo precisa la atribución de recursos y de un tiempo disponible: la consecución de objetivos consume tiempo y recursos. ¿Se tiene claro a qué se renunciará por la consecución del objetivo? ¿A partir de qué momento la persona puede plantearse que ha de renunciar a su objetivo porque ha superado los tiempos prudenciales?

Es necesario incorporar hitos al proceso de consecución del objetivo que permitan identificar a la persona que avanza en la dirección propuesta.

Un objetivo ha de ser compatible con el conjunto de objetivos de la persona: Cuando esto no es así la persona se polariza y aparecen diferentes signos obsesivos. Es necesario, por tanto, ajustar cuidadosamente la jerarquía de objetivos para evitar conflictos entre ellos y prever los efectos (funcionales y disfuncionales) de unos sobre otros.

Los objetivos han ser conocidos por todos los que pudieran estar afectados:

¿quién ha de ser informado?

¿quién puede tener objetivos que choquen con los planteados?

¿cómo se pueden plantear sinergias entre objetivos a fin de maximizar resultados y ahorrar recursos?

No establecer más objetivos que los necesarios, de lo contrario podemos perdernos en una maraña de objetivos difíciles de jerarquizar y conseguir más confusión que claridad. Para identificar los objetivos prioritarios podemos plantear criterios combinados de importancia y urgencia.
Una de las herramientas más útiles para proporcionar un tamiz de realismo a los objetivos es la que se conoce con el nombre de la secuencia de los cómo.

\section{9.- La secuencia de los cómo.}

Se trata de una técnica dirigida a la identificación de recursos, análisis de potencialidades y consecuencias de la aplicación de una idea o decisión a fin de valorar su viabilidad. La técnica se basa en formular hipótesis utilizando sucesivamente la pregunta ¿cómo?

Se puede utilizar para conocer de forma exhaustiva las consecuencias de una decisión e identificar los recursos necesarios para su puesta en marcha y las implicaciones de la misma.

La aplicación de la técnica es relativamente simple en su mecánica. Planteada una pregunta de arranque se trata de preguntar sucesivamente CÓMO, generándose de este modo diversos cursos de acción que pueden ser complementarios o incompatibles entre sí.

La técnica se puede aplicar también en grupo y en contextos organizativos $\mathrm{y}$ resulta especialmente útil cuando los problemas son muy generales en su formulación pero muy complejos en sus implicaciones, por lo que puede necesitar la aportación de diversos profesionales provenientes de distintas áreas.

Se consiguen soluciones realistas a los problemas en la medida que la pregunta ¿cómo? remite inmediatamente a la acción una vez determinados los objetivos.

Se puede utilizar en una enorme variedad de cuestiones desde las más simples a las más complejas en niveles de profundidad discrecionales. 
Cuando se utiliza en contextos organizativos facilita el trabajo conjunto de un equipo de personas sobre un problema determinado.

\section{0.- Identificación previa de los mecanismos de autosabotaje.}

En muchas ocasiones las personas se plantean objetivos y establecen planes de acción muy precisos y técnicamente depurados. Sin embargo ello no es garantía de que los planes se lleven a cabo. Piénsese, por ejemplo, cómo las personas dejan de cumplir con propósitos firmes cuando las circunstancias a las que se enfrentan no son demasiado propicias. Los ejemplos más cotidianos los encontramos en los propósitos relacionados con la salud (dietas, hacer ejercicio, dejar de fumar, etc.) o en los que se relacionan con la conciliación entre el trabajo y la vida social y familiar, entre otras.

La enumeración sistemática y detallada de las razones y circunstancias en las que podemos permitirnos no cumplir con nuestros propósitos actúa como un reforzador de los planes dado que, en alguna medida, desactiva su poder de auto sabotaje.

\section{1.- Suspensión de supuestos.}

Se trata de una técnica planteada por Senge (1982). Cuando se percibe que la otra persona entiende que determinadas condiciones de su situación actual o las personas de su entorno pueden estar dificultando el análisis objetivo de la realidad, o bien sustituyendo aquello que "se quiere" y "desea" por lo que "se debe" o "desean los otros" se puede recurrir a la técnica de la SUSPENSIÓN DE SUPUESTOS. Consiste en invitar al otro a que imagine que los condicionantes no existen: ¿Cuáles serían entonces sus objetivos? ¿Cuáles sus proyectos? ¿De qué manera analizaría la situación actual? ¿Cómo evaluaría su potencial?

La suspensión de supuestos constituye una poderosa técnica para la clarificación de los valores personales y un antídoto para los sesgos en el análisis objetivo de las situaciones actuales que pueden verse enmascaradas por presiones situacionales tanto internas como externas al individuo.

\section{2.- Pregunta sistemática por la causa ¿por qué?}

Dado que en general cuando realizamos atribuciones causales de situaciones o problemas solemos simplificar bastante, es necesario que recuperemos la idea de la complejidad y de la multicausalidad de casi todos los problemas y situaciones de las que nos ocupamos. Casi nada tiene una sola causa. Remontarnos hacia atrás de forma sistemática para encontrar las causas raíces de los efectos que nos preocupan, no sólo nos proporciona una mejor comprensión sino también una cantidad estimable de oportunidades de intervención para modificar los efectos.

\section{3.- Pregunta sistemática por la posibilidad ¿por qué no?}

Puede utilizarse esta pregunta clave para desactivar críticas previas a ideas que rompen los usos y métodos habituales y muy establecidos por la propia experiencia, la rutina o por los contextos sociales en los que participamos.

La técnica ha sido ampliamente utilizada en el mundo del marketing donde ha demostrado sobradamente su utilidad 
para generar innovaciones. Piénsese, por ejemplo, en ¿por qué un banco no puede también vender ordenadores?, ¿por qué una marca de ropa no puede fabricar también artículos de regalo?, ¿por qué un empleado no puede ser también socio?

\section{4.- Distinguir las causas de una situación de los factores que la mantienen.}

No siempre lo que provocó una situación o un déficit determinado es lo que impide que se modifique. Sin embargo, las personas en ocasiones tienden a pensar que las causas mantienen su efecto a través del tiempo y asumen realidades y déficits que, en la práctica, no se encuentran en disposición de continuar activas. Las causas que provocaron un fracaso en una situación determinada pueden haber desaparecido o haberse debilitado en su potencial de actividad; sin embargo lo que mantiene la situación de no enfrentamiento a situaciones similares puede que sea ahora ya, el miedo al fracaso.

\section{7.- Conclusiones}

El desarrollo de las capacidades de liderazgo constituye una de las aplicaciones más relevantes del coaching en los últimos años, sobre todo en el ámbito de las organizaciones laborales. En este sentido, el enfoque de coaching está sustituyendo en gran parte, de forma paulatina, a los antiguos procesos de adquisición de "habilidades para la dirección" y ya no resulta extraño que en las grandes empresas el personal de dirección (incluso mandos intermedios) dispongan de coach personales financiados por las empresas.

Sin embargo, es necesario insistir en dos cuestiones que resultan a nuestro entender esenciales. En primer lugar que el enfoque de coaching no comporta automáticamente un modelo de liderazgo específico sino que puede ser utilizado, y de hecho así está siendo en muchos casos, para reforzar modelos de liderazgo tradicionales a los que aporta mayor eficacia y credibilidad de los líderes en las organizaciones, pero no necesariamente un cambio significativo en la consideración de las personas y de la naturaleza de los roles directivos.

En segundo lugar cabe tener en cuenta que el coaching apenas ha realizado aportaciones de técnicas específicas que puedan considerarse significativas al menos hasta el momento. Sin embargo sí que se trata de un enfoque de trabajo que ha mostrado una gran versatilidad para adaptar técnicas procedentes de distintas tradiciones psicológicas, y del ámbito de la gestión, a procesos de cambio en los que el individuo-cliente cobra un protagonismo extraordinario en comparación con otros enfoques, y que facilita la obtención de cambios relevantes y mantenidos en el tiempo.

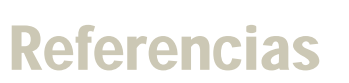

Bass, B.M. (1990): Bass \& Stogdill's Handbook of Leadership. Free Pres. New York.

Bass, B.M. y Avolio, B.J. (1994): Improving Organizational Effectiveness Through Transformational Leadersship. Thousand Oaks, CA: Sage

García Sáiz, M. (2003): El liderazgo en los grupos. En Francisco Gil y Carlos María Alcover (coord. y dir.): Introducción a la psicología de los grupos. Editorial Pirámide, 2003. Pags. $281-330$.

Gaviria, E. (2011): “Visión evolucionista del liderazgo." En F. Molero, y J. Morales (coords.): Liderazgo y: hecho y ficción. Visiones actuales. Pags. 47- 76. Alianza Editorial.

Haslam, S., Reicher, S. y Platow, M. (2011): “El liderazgo como gestión de la identidad social". En F. Molero, y J. 
Morales (coords.): Liderazgo y: hecho y ficción. Visiones actuales. Pags. 77-116. Alianza Editorial.

Hollander, E.P. (1978): Leadership Dynamics. A practical Guide to Effective Relationship. Free Press, New York

House, R. J. y Aditya, R.N. (1997): The social scientific study of leadership: Quo vadis?. Journal of Management, $23,409-473$.

Kets de Vries, M.F.R. y Miller, D. (1984): La organización neurótica. Apóstrofe. Barcelona, 1993

Manz , C.C. y Sims, H.P. (1993): Superliderazgo. Paidós.

Napier, R.V. y Gershenfeld, M.K. (1987): Grupos, teoría y experiencia. Editorial Trillas. México.

Román, J.D. y Fernández, M. (2008): Liderazgo y coaching. Editorial Libros en Red

Senge, P. M.: (1.992) La quinta disciplina. Ediciones Granica.

Tajfel, H. y Turner, J. (1979): "An integrative theory of intergroup conflict" En W.C. Austin y S. Worchel (eds.) The social psychology of intergroup relations. CA: Brooks/Cole. Pags. 33-47. Monterrey.

Van Vugt, M. (2006): “Evolutionary origins of leadership and followership". En Personality and Social Psychology Review, $\mathrm{n}^{0} 10(4)$. pags. 354-371.

Van Vugt, M, Jhonson, D., Kaiser, R., y O'Gorman, R. (2008): Evolution and the social psychology of leadership. The mismatch hypothesis.". En C. Hoyt, D. Forsyth y A. Goethals (eds.) Social Psychology and leadership. Preager. N.Y.

Fecha de recepción: 22/10/2013

Fecha de aceptación: 1/04/2014 